\title{
The Design and Application of A Novel Duplex Coating Formed by Laser Pre-treatment
}

\author{
Kun Zhang ${ }^{1, a}$, Chen-Wu Wu ${ }^{1,2, b}$, Ying Hu${ }^{1,2, c}$, Guang-Nan Chen ${ }^{1, d}$ \\ ${ }^{1}$ Materials Processing Center, Institute of Mechanics, Chinese Academy of Sciences, \\ Beijing100080, China \\ ${ }^{2}$ Graduate School of the Chinese Academy of Sciences, Beijing100080, China \\ akzhang@imech.ac.cn, 'bcw_on@yahoo.com.cn, hying320@126.com, dgnchen@imech.ac.cn
}

Keywords: duplex coating, laser, discrete quenching, mesostructure, tempering

\begin{abstract}
A novel duplex coating is presented on steel substrate by laser discrete quenching prior to chromium coating deposition. Due to the periodical changes in the mesostructures and properties of underlying substrate, the cracking and spalling behaviors of the duplex coating are expected to be different from that of conventional coating. A classic erosion experiment is selected to characterize the positive response of the duplex coating subjected to thermal loads. It is found that the surface cracking is partly restrained and the interface cracking is partly arrested. FEM modeling results show that a unique stress state is induced in the duplex coating with the tempering of the discrete pre-quenched regions due to thermal loading, which is believed to be the mechanism of controlling the damage modes.
\end{abstract}

\section{Introduction}

The technique of laser discrete quenching without the overlapping of individual laser beam tracks corresponding to the width of the laser beam has been widely accepted for iron-based alloys. In the case of the production of wear resistance surface, the special surface mesostructures, i.e. the co-existence of quenched (harder) and unquenched (relatively softer) regions may be advantage in terms of the entrapment of wear debris. Besides, H. Shen et al [1] reported that the plastic stability of a steel sheet is improved after laser indirect texturing, because the discrete prestrained microcraters enforced on the surface of the textured sheet act as hardening spots, which are likely to share out deformation and inhibit the increasing rate of voiding. Such concepts as discrete mesostructures allure one to extend them to other fields in materials engineering.

Coatings with resistance to wear, corrosion and thermal degradation are adopted in a great many fields. Unfortunately, the prospective applications of coatings are restricted with its premature spalling. Many designs such as gradient coating, multilayer coating as well as duplex coating [2-4] are developed to enhance the durability of coating. These designs are commonly based on a homogeneous coating and substrate along the interface, i.e. the arrangements of materials vary perpendicular to the interface. In this study, the technique of laser discrete pre-treatment is used to form a novel duplex coating, in which the properties and states of substrate alter periodically along the interface. It is expected that the cracking and spalling behavior of this coating is different from that of conventional coating. For a preliminary study, laser scanning quenching is adopted to achieve a one-dimensional discrete substrate rather than two-dimensional one, the latter of which can be realized by laser spot quenching prior to coating deposition. Aimed at a model materials pair, thermal loading experiment and finite element modeling (FEM) are used to reveal the response of 
duplex coating. Fortunately, some inspired results arise including restraining of surface cracking and arresting of interface cracking.

\section{Experimental}

A low-carbon alloy steel (30CrNi2MoVA) is adopted as the substrate material. Prior to chromium coating electrodeposition, laser quenching treatment is performed using a CW Nd:YAG laser without the overlapping of individual tracks. The final specimen is shown in Fig.1. In such a duplex coating, the discrete quenched regions distribute periodically along the interface between the coating and the substrate.

A classic erosion experiment in bore [5], in which chromium coating often suffers from spalling, is used to characterize the response of the duplex coating. Such an experiment as gun firing can provide distinctive temperature levels along the axis of bore simultaneously, which is useful for observing the evolution of damage patterns in the coating with almost identical processing parameters. In general, the peak value of temperature decreases from breech to muzzle. After being heated for defined cycles, three specimens from typical longitudinal locations along the axis of bore are metallographically prepared to analyze the damage patterns.

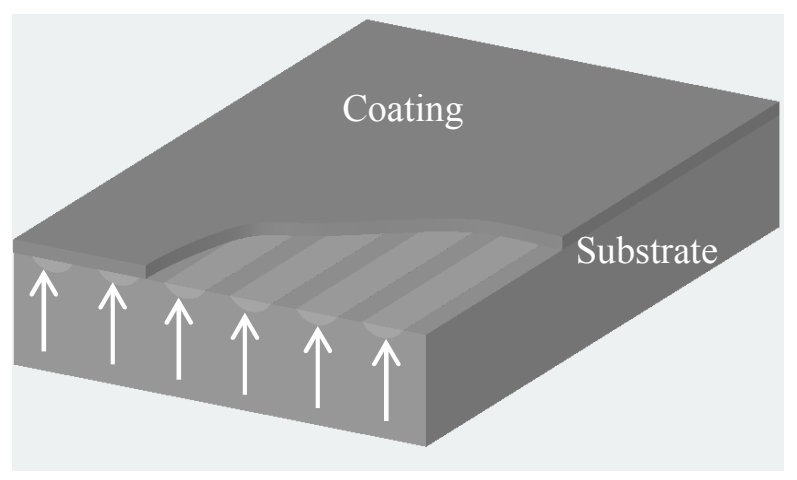

(a) Sketch

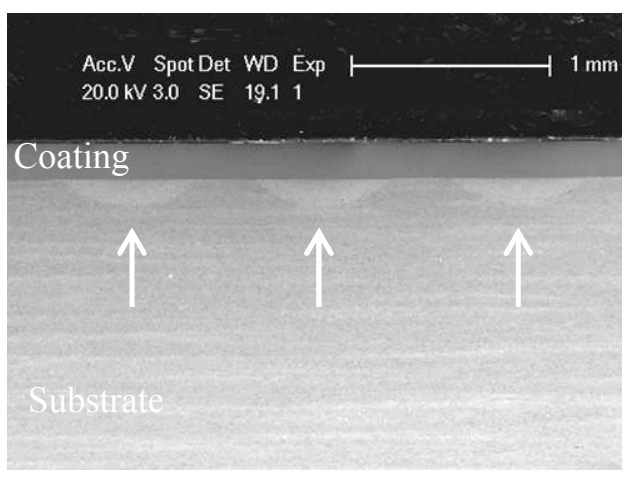

(b) Metallography

Fig.1. Mesostructure of the duplex coating (The discrete quenched regions are directed with white arrows)

\section{Experimental Results and Modelling}

Fig. 2 shows the damage patterns of the structure, where the specimen in each upper photo is polished to reveal cracks, whereas underside one is etched to reveal the profiles of the discrete quenched regions. Figs.2 (a), (b) and (c) correspond to temperature level of 1st, 2nd and 3rd, which represent the lower, medium and higher temperature, respectively. It can be found that the surface cracking develops mostly within the space between two quenched regions, and the surface cracking of the coating adherent to the quenched regions is restrained in a large scale. This effect is more obvious in Figs.2 (a) and (b). The surface cracks propagate deeply with the increase of temperature level and those impinging upon the interface are known as segmentation cracks. Interface cracking has a tendency to develop around the tips of segmentation cracks. Surprisingly, the interface cracking, apparent in Fig.2 (c) but dim in Fig.2 (b), is partitioned and arrested within the space between two adjacent quenched regions. This effect is considered to be positive to improve spalling resistance of coating, for spalling will not occur until interfacial crack is intersected with another segmentation crack. 

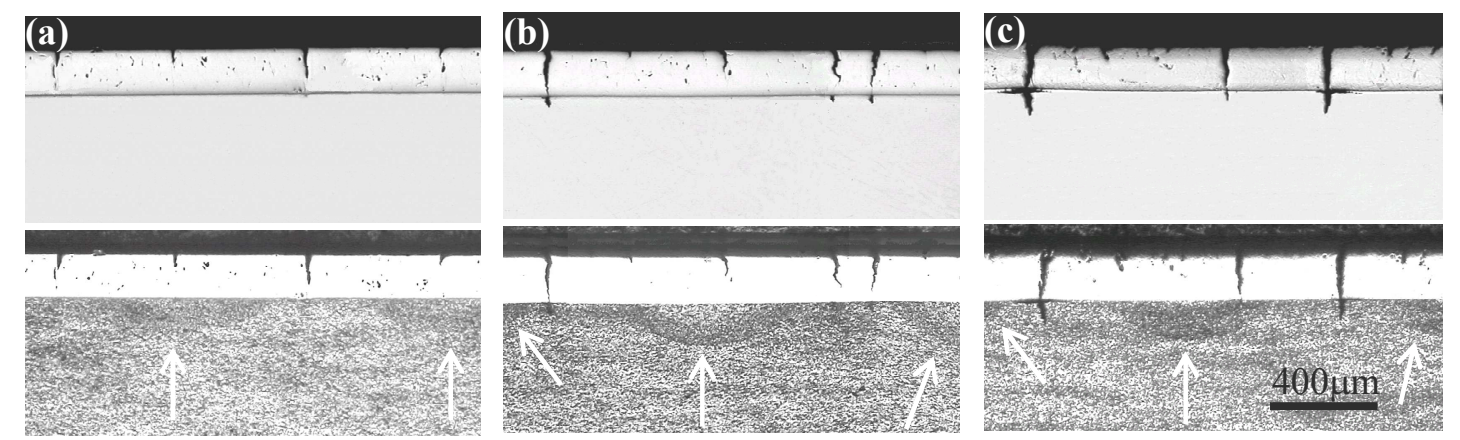

Fig.2. Metallography of coating damage at temperature level of (a) 1st, (b) 2 nd and (c) $3^{\text {rd }}$

To reveal the mechanisms, the duplex coating is modeled as shown in Fig.3, where $R$ is the width of the quenched region, $S$ the space between two quenched regions, $W=R+S$ the period of the mesostructure and $D$ the thickness of chromium coating, which is also approximately the depth of quenched region. The discrete quenched regions will be tempered and contract after being heated for several cycles of thermal loads. Correspondingly, internal stresses will develop within the coating and substrate, which is simulated with FEM and the results are shown in Fig.4 and Fig.5. Note that $\sigma_{0}=E^{*} \times \varepsilon_{0}$, where $E^{*}=E_{\mathrm{c}} E_{\mathrm{s}} /\left(E_{\mathrm{c}}+E_{\mathrm{s}}\right)$, in which $E_{\mathrm{c}}$ and $E_{\mathrm{s}}$ represent Young's Modulus of coating and substrate respectively, and $\varepsilon_{0}$ is defined as eigen strain corresponding to average dilation coefficient during phase transformation. In this study, the dimensional parameters are chosen as $R=700 \mu \mathrm{m}, S=300 \mu \mathrm{m}$ and $D=190 \mu \mathrm{m}$, material parameters $E_{c}=300 \mathrm{GPa}, E_{s}=200 \mathrm{GPa}$, $v_{c}=0.2, v_{s}=0.3$ and $\varepsilon_{0}=0.0028[6]$, here $v_{c}$ and $v_{s}$ is Poisson's ratio of coating and substrate respectively. All deformations are assumed to be elastic in the calculations.

Fig. 4 shows that the internal stresses $\sigma_{x x}$ in coating alter periodically corresponding to the period of the discrete quenched regions, which are mainly tensile in the portions of coating adherent to unquenched regions but compressive in those adherent to quenched regions. This is in accordance with the coating cracking modes, i.e. the surface cracking develops mostly within the space between two quenched regions. Fig.5 shows the stresses $\sigma_{y y}$ along the vertical path (The path is shown in Fig.3), and the stresses remain compressive. Such compressive stresses $\sigma_{y y}$ will contribute negatively to the synthetical stress intensity factor of a crack [7], which maybe a mechanism restraining and partitioning the interface cracking.

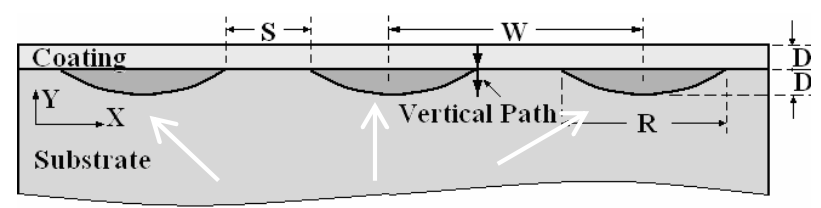

Fig.3. Model of the duplex coating

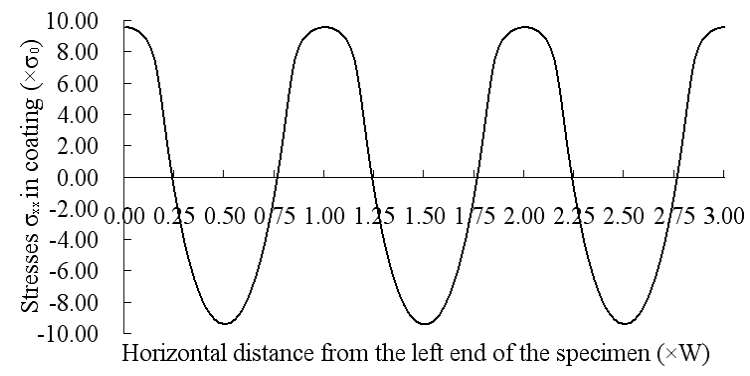

Fig.4. Stresses in the coating

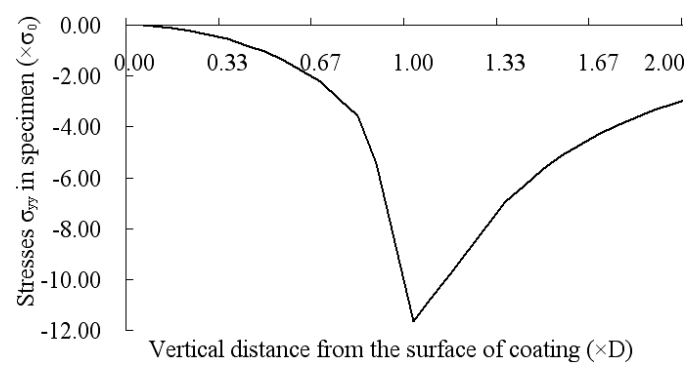

Fig.5. Stresses along vertical path 


\section{Conclusions}

A duplex coating is attained with laser pre-treatment and electroplating chromium, in which the substrate possesses discrete quenched regions along the interface. The thermal damage patterns of coating are controlled by such a periodical mesostructures, i.e., the coating cracking and interface cracking are corresponding to the periods of the structure. Furthermore, the interface cracking is partitioned and arrested within the space between two adjacent quenched regions. Modeling analysis and calculations indicate that the tempering of the periodic pre-quenched regions induce a unique internal stresses state within coating and substrate, of which the periodical tensile stresses in the coating will lead to the periodical cracking of coating while the periodical compressive stresses perpendicular to the interface may partition the interface cracking.

\section{Acknowledgements}

The financial support provided by the National Natural Science Foundation of China (Grant NO. 50471087 and 50531060) is greatly acknowledged.

\section{References}

[1] H. Shen, G.N. Chen, G.C. Li, Mater. Sci. Eng., A, Vol. A219 (1996), P. 156

[2] D. Kim, M. Kim, K.S. Nam, D. Chang and S.C. Kwon, Surf. Coat. Technol., Vol. 169-170 (2003), P. 650

[3] C. Pfohl, K.-T. Rie, Surf. Coat. Technol., Vol. 142-144 (2001), P. 1116

[4] T. Bell, H. Dong and Y. Sun, Tribol. Int., Vol. 31 (1998), P. 127

[5] S. Sopok, C. Rickard, S. Dunn, Wear, Vol. 258 (2005), P. 659

[6] Z. Liu, Z.J. Wu, J.Z. Wu, Y. Zhang: Numerical Simulation on Heat Treatment Process (Science Press, China 1996).

[7] M. Lugovy, V. Slyunyayev, V. Subbotin, N. Orlovskaya, G. Gogotsi, Compos. Sci. Technol., Vol. 64 (2004), P. 1947 


\section{Heat Treatment of Materials}

10.4028/www.scientific.net/SSP.118

\section{The Design and Application of a Novel Duplex Coating Formed by Laser Pre-Treatment}

10.4028/www.scientific.net/SSP.118.243

\section{DOI References}

[5] S. Sopok, C. Rickard, S. Dunn, Wear, Vol. 258 (2005), P. 659

doi:10.1016/j.wear.2004.09.031

[7] M. Lugovy, V. Slyunyayev, V. Subbotin, N. Orlovskaya, G. Gogotsi, Compos. Sci. Technol., Vol. 64 (2004), P. 1947

doi:10.1016/j.compscitech.2004.02.007 